Pacific Journal of Mathematic 


\title{
ON THE DISTRIBUTION OF PYTHAGOREAN TRIANGLES
}

\author{
J. LAMBEK AND L. MOSER
}

1. Introduction. This paper was conceived with the object of estimating the number $P_{a}(n)$ of primitive Pythagorean triangles with area less than $n$. The problem seemed of interest, since F. L. Miksa [5] recently tabulated all primitive Pythagorean triangles with area less than $10^{9}$, in order of increasing area. The method employed here also yields known estimates for the numbers $P_{h}(n)$ and $P_{p}(n)$ of primitive Pythagorean triangles with hypotenuse and perimeter, respectively, less than $n$; we use $P(n)$ as generic notation for all of these.

D. N. Lehmer [4] had shown in 1900 that

$$
P_{h}(n) \sim \frac{1}{2} \pi^{-1} n, \quad P_{p}(n) \sim \log 2 \cdot \pi^{-2} n
$$

In 1948, D. H. Lehmer [3] obtained

$$
P_{p}(n)=\log 2 \cdot \pi^{-2} n+O\left(n^{1 / 2} \log n\right),
$$

pointing out that this disproved a conjecture of Krishnaswami [2] that $P_{p}(n) \sim$ $n / 7$. For primitive Pythagorean triangles with area less than $2.10^{6}$, W. P. Whitlock [6] found that

$$
\left|P_{a}(n)-\frac{1}{2} n^{1 / 2}+5\right| \leq 2
$$

However, Miksa's table, which goes 500 times as far as Whitlock's, suggested that $P_{a}(n)$ is not asymptotic to $(1 / 2) n^{1 / 2}$.

In $\& 2$ we reduce the problem of approximating $P(n)$ to that of estimating the number of lattice points in certain regions of the Cartesian plane. The latter problem is treated in $\xi 3$, with some attempt at generality. In $\S 4$ we obtain the following asymptotic formulae for $P(n)$ :

$$
P_{h}(n)=\frac{1}{2} \pi^{-1} n+O\left(n^{1 / 2} \log n\right)
$$

Received June 16, 1953. This paper was written at the Summer Research Institute of the Canadian Mathematical Congress.

Pacific J. Math., 5 (1955), $73-83$ 


$$
\begin{aligned}
& P_{p}(n)=\log 2 \cdot \pi^{-2} n+O\left(n^{1 / 2} \log n\right), \\
& P_{a}(n)=c n^{1 / 2}+O\left(n^{1 / 3}\right),
\end{aligned}
$$

where $c=\Gamma(1 / 4)^{2} 2^{-1 / 2} \pi^{-5 / 2}=.531340 \ldots$.

Let $E(n)=c n^{1 / 2}-P_{a}(n)$. The following table, constructed on the basis of Miksa's tabulations, gives an idea of the possible constant suggested by $E(n)=$ $O\left(n^{1 / 3}\right)$ :

\begin{tabular}{ccccc}
$10^{-8} n$ & $P_{a}(n)$ & $c n^{1 / 2}$ & $E(n)$ & $E(n) n^{-1 / 3}$ \\
\hline 1 & 5157 & 5313.4 & 138 & .298 \\
2 & 7342 & 7514.3 & 172 & .294 \\
3 & 9007 & 9203.1 & 196 & .295 \\
4 & 10405 & 10627 & 222 & .301 \\
5 & 11644 & 11908 & 237 & .299 \\
6 & 12778 & 13015 & 237 & .282 \\
7 & 13800 & 14058 & 258 & .291 \\
8 & 14755 & 15029 & 274 & .296 \\
9 & 15655 & 15940 & 285 & .295 \\
10 & 16513 & 16802 & 289 & .289 \\
\hline
\end{tabular}

For the foregoing data, the average value of $E(n) n^{-1 / 3}$ is .295 with a standard deviation of .00405 . We are led to conjecture that $E(n) \sim c^{\prime} n^{1 / 3}$, where $c^{\prime}$ is approximately .295 .

2. Pythagorean triangles. A Pythagorean triangle

$$
\Delta=\langle a, b, c\rangle=\langle b, a, c\rangle
$$

is determined by three positive integers $a, b, c$ such that $a^{2}+b^{2}=c^{2}$. If their greatest common divisor $(a, b, c)=1$, then $\Delta$ is called primitive. If $(a, b, c) \leq 2$, we shall call $\Delta$ quasi-primitive. An integral lattice point $\langle x, y\rangle$ on the Cartesian plane will be called primitive if $(x, y)=1$.

Lemma 1. The equations

$$
a=2 x y, \quad b=x^{2}-y^{2}, \quad c=x^{2}+y^{2}
$$

determine a one-to-one correspondence between all quasi-primitive Pythagorean 
triangles $\Delta=\langle a, b, c\rangle$ and all primitive lattice points $\langle x, y\rangle$ of the region $x>y>0$ of the Cartesian plane.

Proof. If $a, b, c$ are given by (1), then clearly $\Delta$ is a Pythagorean triangle. Moreover, if $(x, y)=1, \Delta$ will be quasi-primitive. It is known $[1, p .189]$ that all primitive Pythagorean triangles are uniquely expressible in the form ( 1 ) with $(x, y)=1$ and $x \neq \equiv y(\bmod 2)$. It remains to consider the case $(a, b, c)=2$. Then $\langle a / 2, b / 2, c / 2\rangle$ is primitive, and we may write

$$
a / 2=x^{\prime 2}-y^{\prime 2}, \quad b / 2=2 x^{\prime} y^{\prime}, \quad c / 2=x^{\prime 2}+y^{\prime 2},
$$

where

$$
x^{\prime}>y^{\prime}>0,\left(x^{\prime}, y^{\prime}\right)=1, \quad x^{\prime} \not \equiv y^{\prime}(\bmod 2) .
$$

If we now let $x=x^{\prime}+y^{\prime}, y=x^{\prime}-y^{\prime}$ and eliminate $x^{\prime}, y^{\prime}$ in favor of $x, y$, we may easily verify (1) and $x>y>0,(x, y)=1$. This completes the proof.

In the following, let $F(\Delta)=F(a, b, c)$ be homogeneous of degree $k>0$ in $a, b, c$, such that there are only finitely many $\Delta$ with $F(\Delta)<n$. Without loss in generality we may assume that $F$ has been normalized so that

$$
F(\Delta) \geq 1
$$

In this paper, we are interested in three special cases:

Case 1. $F(\Delta)=c$ (hypotenuse), $k=1$.

Case 2. $F(\Delta)=a+b+c$ (perimeter), $k=1$.

Case 3. $F(\Delta)=a b / 2$ (area), $k=2$.

It is seen that Condition (2) is satisfied in these cases.

We wish to find the number $P(n)$ of primitive and the number $Q(n)$ of quasiprimitive Pythagorean triangles $\Delta$ for which $F(\Delta)<n$. Now $F(2 a, 2 b, 2 c)<n$ if and only if $F(a, b, c)<n / 2^{k}$; hence

$$
Q(n)=P(n)+P\left(n / 2^{k}\right) .
$$

This formula may be inverted to give

$$
P(n)=\sum_{i \geq 0}(-1)^{i} Q\left(n / 2^{k i}\right)
$$


It should be noted that this is a finite sum, since, by (2), $Q(1)=0$.

The calculation of $P(n)$ is thus reduced to that of $Q(n)$. By Lemma 1 , $Q(n)$ is the number of primitive lattice points in the region of the Cartesian plane defined by the inequalities

$$
G(x, y)=F\left(2 x y, x^{2}-y^{2}, x^{2}+y^{2}\right)<n, x>y>0 .
$$

If we write $n=t^{2 k}$, this is the same as the region

$$
G(x / t, y / t)<1, x>y>0,
$$

that is, the set of all points $\langle x, y\rangle$ for which $\langle x / t, y / t\rangle$ lies in the region $R$ defined by

$$
G(X, Y)<1, X>Y>0 \text {. }
$$

If $R$ is any subset of the Cartesian plane, and $t$ any positive real number, we define $R t$ to be the region obtained from $R$ by radial magnification in the ratio $t: 1$, so that $\langle x, y\rangle$ lies in $R t$ if and only if $\langle x / t, y / t\rangle$ lies in $R$. Furthermore, let $L(R)$ denote the number of integral lattice points in $R, L^{\prime}(R)$ the number of primitive lattice points in $R$.

In particular, if $R$ is the region defined by (5), it follows that (4) is the region $R t$, so that

$$
Q(n)=L^{\prime}(R t), n=t^{2 k} \text {. }
$$

For any $R,\langle x, y\rangle$ is an integral lattice point in $R t$ with $(x, y)=i$ if and only if $\langle x / i, y / i\rangle$ is a primitive lattice point in $R t / i$. Hence

$$
L(R t)=\sum_{i \geq 1} L^{\prime}(R t / i)
$$

To avoid questions of convergence, we shall confine our attention to the case

$$
L(R)=0, L^{\prime}(R)=0,
$$

these two conditions being clearly equivalent. In particular, if $R$ is the region defined by (5), then (8) follows from (2). The expression on the right of (7) is now a finite sum, and may be inverted with the help of the well-known Möbius function $\mu(i)[1$, p. 236] to give 


$$
L^{\cdot}(R t)=\sum_{i \geq 1} \mu(i) L(R t / i)
$$

This again is a finite sum.

In view of (3), (6), and (9), the problem of calculating $P(n)$ has been reduced to that of counting the number of lattice points in the region $R t$, where $R$ is given by ( 5 ).

3. On the number of lattice points in a region. Let $R$ be an open set in the Cartesian plane. We wish to approximate the number $L(R t)$ of integral lattice points in $R t$ by the measure

$$
M(R t)=M(R) t^{2}
$$

of $R t$. Here, as before, $R t$ denotes the region obtained from $R$ by radial magnification in the ratio $t: 1$.

Instead of fixing the lattice and magnifying $R$ in the ratio $t: 1$, we may keep $R$ fixed and shrink the mesh of the lattice in the ratio $1: t$. Let $L_{t}$ denote the lattice thus contracted, with mesh length $1 / t$. Then $L(R t)$ is also the number of vertices of $L_{t}$ in $R$.

Lemm A 2. If $R$ is the open region enclosed by a simple closed Jordan curve in the Cartesian plane, whose total horizontal plus vertical variation is $V$, then

$$
|L(R t)-M(R t)| \leq V t
$$

Proof. Let $L_{t}^{*}$ be the lattice conjugate to $L_{t}$, that is, the square lattice whose vertices are the midpoints of the squares of $L_{t}$. Then each vertex of $L_{t}$ in $R$ lies in a square of $L_{t}^{*}$ which has a part in common with $R$, and each square of $L_{t}^{*}$ contained in $R$ contains a vertex of $L_{t}$. Let $s_{t}(R)$ denote the number of closed squares of $L_{t}^{*}$ contained in $R$, and $S_{t}(R)$ the number of open squares of $L_{t}^{*}$ having a part in common with $R$; then

$$
s_{t}(R) \leq L(R t) \leq S_{t}(R) .
$$

Moreover, comparing areas, we obtain

$$
s_{t}(R) t^{-2} \leq M(R) \leq S_{t}(R) t^{-2},
$$

so that 


$$
\left|L(R t)-M(R) t^{2}\right| \leq S_{t}(R)-s_{t}(R) .
$$

Now this is the number of open squares of $L_{t}^{*}$ which contain portions of the given Jordan curve $J$, hence does not exceed the number of horizontal and vertical lines of $L_{t}^{*}$ crossed by $J$. As there are $t$ mesh lengths of $L_{t}^{*}$ per unit interval, the latter number is bounded by $V t$, where $V$ is the total horizontal plus vertical variation of $J$. This completes the proof.

We wish to obtain a result analogous to Lemma 2 for unbounded regions. It seems difficult to state the most general result of this kind. Here we confine our attention to the following:

Lemma 3. Let $R$ be the region in the Cartesian plane defined in polar coordinates $\rho, \vartheta$ by the inequalities

$$
0<\rho<f(\vartheta), 0 \leq \alpha<\vartheta<\beta \leq \pi / 2,
$$

subject to:

(i) $f(\vartheta)$ is continuous, increasing, and positive for $\alpha \leq \vartheta<\beta$,

(ii) $f(\vartheta) \asymp(\beta-\vartheta)^{\mu-1^{*}}, 1>\mu>1 / 2$,

(iii) $\tan \beta$ is rational.

Then

$$
L(R t)-M(R t)=O\left(t^{1 / \mu}\right) .
$$

Proof. The distance from a point on the curve $\rho=f(\vartheta)$ to the line $\vartheta=\beta$ is given by

$$
g(\vartheta)=f(\vartheta) \sin (\beta-\vartheta) \asymp(\beta-\vartheta)^{\mu},
$$

which tends to 0 as $\vartheta \rightarrow \beta$, since $\mu \geq 0$. On the other hand,

$$
f(\vartheta) \asymp(\beta-\vartheta)^{\mu-1}
$$

tends to infinity, since $\mu<1$. Hence the line $\vartheta=\beta$ is an asymptote of the curve.

We shall write

$$
\tan \beta=p / q,(p, q)=1, p^{2}+q^{2}=r^{2} \text {. }
$$

The distance from a point $\langle x, y\rangle$ below the asymptote to the asymptote is then

* The symbol of $f \asymp g$ is used to denote $0<\underline{\lim } f / g \leq \lim _{f} / g<\infty$. 


$$
\rho \sin (\beta-\vartheta)=x \sin \beta-y \cos \beta=(p x-q y) / r
$$

Hence the smallest nonzero distance which any integral lattice point can have from the asymptote is $1 / r$, and the smallest nonzero distance from a vertex of $L_{t}$ to the asymptote is $1 /(r t)$.

For sufficiently large $t$, we have

$$
g(\alpha)>\frac{1}{2 r t}
$$

Since $g(\vartheta) \longrightarrow 0$, we can choose a $\vartheta_{t}$ such that

$$
g\left(\vartheta_{t}\right)=f\left(\vartheta_{t}\right) \sin \left(\beta-\vartheta_{t}\right)=\frac{1}{2 r t}
$$

and $g(\vartheta)<1 /(2 r t)$ for $\vartheta>\vartheta_{t}$. Let $R_{t}$ be the region defined by

$$
0<\rho<f(\vartheta), \vartheta_{t} \leq \vartheta<\beta
$$

then $R_{t}$ contains no vertices of $L_{t}$; that is, $L\left(R_{t} t\right)=0$. Hence

$$
L(R t)-M\left(R_{t}\right)=L\left(R t-R_{t} t\right)-M\left(R_{t}-R_{t} t\right)-M\left(R_{t} t\right),
$$

so that

$$
|L(R t)-M(R t)| \leq V_{t} t+M\left(R_{t} t\right)
$$

by Lemma 2, if $V_{t}$ denotes the total (horizontal plus vertical) variation of the boundary of $R-R_{t}$. It remains to estimate $V_{t} t$ and $M\left(R_{t} t\right)$.

We claim that $V_{t}=O\left(f\left(\vartheta_{t}\right)\right)$. For the boundary of $R-R_{t}$ consists of two straight segments of lengths $f(\alpha)$ and $f\left(\vartheta_{t}\right)$ and the $\operatorname{arc} \rho=f(\vartheta), \alpha<\vartheta<\vartheta_{t}$. We need only consider the variation of the latter. Its vertical variation is the variation of $f(\vartheta) \sin \vartheta$. Now this is an increasing function of $\vartheta$, and hence has variation

$$
O\left(f\left(\vartheta_{t}\right) \sin \vartheta_{t}\right)=O\left(f\left(\vartheta_{t}\right)\right)
$$

The horizontal variation of the arc is the variation of $f(\vartheta) \cos \vartheta$. Now this can be expressed as the difference of two increasing functions $f(\vartheta)$ and $f(\vartheta)(1-$ $\cos \vartheta$ ), both of whose variations are $O\left(f\left(\vartheta_{t}\right)\right)$. Hence so is the horizontal variation and therefore also the total variation of the arc, as was to be proved. 
From (10) and (11) we obtain

$$
\left(\beta-\vartheta_{t}\right)^{\mu} \asymp t^{-1}
$$

hence

$$
\left.V_{t} t=O\left(f\left(\vartheta_{t}\right)\left(\beta-\vartheta_{t}\right)^{-\mu}\right)=O\left(\beta-\vartheta_{t}\right)^{-1}\right)=O\left(t^{1 / \mu}\right),
$$

as required. Finally,

$$
M\left(R_{t} t\right)=M\left(R_{t}\right) t^{2}=\frac{1}{2} t^{2} \int_{\vartheta}^{\beta} f(\vartheta)^{2} d \vartheta=O\left(t^{2} \int_{\vartheta}^{\beta}(\beta-\vartheta)^{2 \mu-2} d \vartheta\right),
$$

by (ii). Since $\mu>1 / 2$, this is

$$
O\left(\left(\beta-\vartheta_{t}\right)^{2 \mu-1} t^{2}\right)=O\left(\left(\beta-\vartheta_{t}\right)^{-1}\right)=O\left(t^{1 / \mu}\right),
$$

by (13). In view of (12), this completes the proof of Lemma 3.

4. Distribution of Pythagorean triangles. We shall obtain asymptotic formulae for $Q(n)$ and $P(n)$ in the three cases under consideration.

Case 1. Estimation of $P_{h}(n)$. Here $F(a, b, c)=c, k=1$; and $R$ is given by

$$
x^{2}+y^{2}<1, x>y>0
$$

Clearly $M(R)=\pi / 8$. Lemma 2 yields

$$
L(R t)=M(R) t^{2}+O(t)
$$

Hence, by (9),

$$
\begin{aligned}
L^{\prime}(R t) & =\sum_{1 \leq i \leq t} \mu(i) L(R t / i) \\
& =\sum_{1 \leq i \leq t}\left\{\mu(i) M(R)(t / i)^{2}+O(t / i)\right\} \\
& =M(R) t^{2}\left\{6 \pi^{-2}+O\left(t^{-1}\right)\right\}+O(t \log t) \\
& =\frac{3}{4} \pi^{-1} t^{2}+O(t \log t) .
\end{aligned}
$$


Then ( 6 ) becomes

$$
Q_{h}(n)=\frac{3}{4} \pi^{-1} n+O\left(n^{1 / 2} \log n\right)
$$

and (3) gives rise to

$$
\begin{aligned}
P_{h}(n) & =\sum_{i \geq 0}(-1)^{i} Q\left(n / 2^{i}\right) \\
& =\frac{3}{4} \pi^{-1} n \sum_{i \geq 0}(-1 / 2)^{i}+O\left(\sum_{i \geq 0}\left(n / 2^{i}\right)^{1 / 2} \log \left(n / 2^{i}\right)\right) \\
& =\frac{1}{2} \pi^{-1} n+O\left(n^{1 / 2} \log n\right),
\end{aligned}
$$

as stated in $\& 1$.

Case 2. Estimation of $P_{p}(n)$. Here $F(a, b, c)=a+b+c, k=1$, and $R$ is given by

$$
2 x(x+y)<1, x>y>0 .
$$

By integration, $M(R)=(\log 2) / 4$. Calculating as in Case 1 , we obtain

$$
Q_{p}(n)=\frac{3}{2} \log 2 \cdot \pi^{-2} n+O\left(n^{1 / 2} \log n\right),
$$

and

$$
P_{p}(n)=\log 2 \cdot \pi^{-2} n+O\left(n^{1 / 2} \log n\right),
$$

as stated in $\S 1$.

Case 3. Estimation of $P_{a}(n)$. Here $F(a, b, c)=a b / 2, k=2$; and $R$ is given by

$$
x y\left(x^{2}-y^{2}\right)<1, x>y>0 .
$$

Transformed into polar coordinates, this becomes

$$
\rho^{4} \sin 4 \vartheta<4,0<\vartheta<\pi / 4 \text {. }
$$


By integration,

$$
M(R)=2^{-5 / 2} \pi^{-1 / 2} \Gamma(1 / 4)^{2}
$$

The line $\vartheta=\pi / 8$ separates $R$ into two subregions $R_{1}$ and $R_{2}$, which we shall take to be open sets, $R_{1}$ with asymptote $\vartheta=0$, and $R_{2}$ with asymptote $\vartheta=\pi / 4$. $R_{2}$ satisfies the conditions of Lemma 3 , with $\mu=3 / 4$. Although Lemma 3 does not apply directly to $R_{1}$, it may be used for the reflection of $R_{1}$ about the line $\vartheta=\pi / 4$. Such a reflection does not affect the area of $R_{1}$ or the number of lattice points in it. Again we have $\mu=3 / 4$. Hence

$$
L\left(R_{i} t\right)=M\left(R_{i} t\right)+O\left(t^{4 / 3}\right) \quad(i=1,2)
$$

Adding these two equations, and observing that there are no vertices of $L_{t}$ on the dividing line $\vartheta=\pi / 8$, its slope being irrational, we obtain

$$
L(R t)=M(R) t^{2}+O\left(t^{4 / 3}\right) .
$$

Hence, by (9),

$$
\begin{aligned}
L^{\prime}(R t) & =\sum_{i \geq 1} \mu(i) L(R t / i) \\
& =\sum_{i \geq 1}\left\{\mu(i) M(R)(t / i)^{2}+O(t / i)^{4 / 3}\right\} \\
& =6 \pi^{-2} M(R) t^{2}+O\left(t^{4 / 3}\right) .
\end{aligned}
$$

Then (6) becomes

$$
Q_{a}(n)=6 \pi^{-2} M(R) n^{1 / 2}+O\left(n^{1 / 3}\right),
$$

so that, by (3),

$$
\begin{aligned}
P_{a}(n) & =\sum_{i \geq 0}(-1)^{i} Q\left(n / 4^{i}\right) \\
& =6 \pi^{-2} M(R) n^{1 / 2} \sum_{i \geq 0}(-1 / 2)^{i}+O\left(\sum_{i \geq 0}\left(n / 4^{i}\right)^{1 / 3}\right) \\
& =4 \pi^{-2} M(R) n^{1 / 2}+O\left(n^{1 / 3}\right) .
\end{aligned}
$$


Replacing $M(K)$ by its numerical value, we obtain the result stated in $\delta 1$.

\section{REFERENCES}

1. G. H. Hardy and E. M. Wright, An introduction to the theory of numbers, Oxford, 1945.

2. A. A. Krishnaswami, On is operimetrical Pythagorean triangles, Tôhoku Math. J. 27 (1926), $332-348$.

3. D. H. Lehmer, A conjecture of Krishnaswami, Bull. Amer. Math. Soc. 54 (1948), $1185-1190$.

4. D. N. Lehmer, Asymptotic evaluation of certain totient sums, Amer. J. Math. 22 (1900), 293 - 335.

5. F. L. Miksa, Table of primitive Pythagorean triangles with area up to $10^{9}$ in order of increasing area, unpublished.

6. W. P. Whit lock Jr., Rational right triangles with equal area, Scripta Math.9 (1943), $155-167$ and 265-268.

MCGILL UNIVERSITY,

UNIVERSITY OF ALBERTA 




\section{Pacific Journal of Mathematics}

\section{Vol. 5, No. $1 \quad$ September, 1955}

Frank Herbert Brownell, III, Flows and noncommuting projections on

Hilbert space ................................... 1

H. E. Chrestenson, A class of generalized Walsh functions ............. 17

Jean Bronfenbrenner Crockett and Herman Chernoff, Gradient methods of maximization ................................... 33

Nathan Jacob Fine, On groups of orthonormal functions. I .......... 51

Nathan Jacob Fine, On groups of orthonormal functions. II ............ 61

Frederick William Gehring, A note on a paper by L. C. Young .......... 67

Joachim Lambek and Leo Moser, On the distribution of Pythagorean

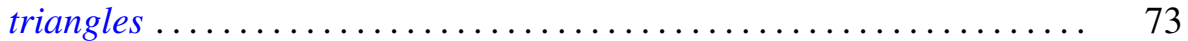

Roy Edwin Wild, On the number of primitive Pythagorean triangles with

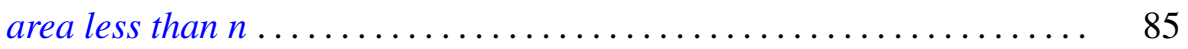

R. Sherman Lehman, Approximation of improper integrals by sums over multiples of irrational numbers ........................ 93

Emma Lehmer, On the number of solutions of $u^{k}+D \equiv w^{2}(\bmod p) \ldots 103$

Robert Delmer Stalley, A modified Schnirelmann density............... 119

Richard Allan Moore, The behavior of solutions of a linear differential equation of second order............................. 125

William M. Whyburn, A nonlinear boundary value problem for second order differential systems. 